\title{
JOT JOURNAL OF TURBULENCE
}

http://jot.iop.org/

\section{Modification of near-wall turbulence due to spanwise wall oscillations}

\author{
Pierre Ricco ${ }^{1}$ \\ Mechanical Engineering Department, The University of Texas at Austin, \\ 1 University Station C2200, Austin, TX 78712, USA \\ E-mail: pierre.ricco@imperial.ac.uk
}

Received 16 February 2004

Published 9 July 2004

doi:10.1088/1468-5248/5/1/024

\begin{abstract}
An experimental investigation of a turbulent boundary layer modified by spanwise wall oscillations is conducted in a water channel by means of the hydrogen-bubble technique. The purpose is to study the dynamics of near-wall turbulent structures to shed new light on the physical mechanisms characterizing the boundary layer perturbed by the wall motion and to comprehend how these changes cause a wall-shear stress reduction. It is likely that flow visualizations conducted at the highest values of maximum wall velocity describe the spatial transient evolution of the flow to the new modified state because of the limited extension of the oscillating wall section. When the oscillatory motion is imposed, the low-speed streaks shift laterally, and cyclically incline to an angle with respect to the streamwise direction. Flow visualizations from the end of the water channel distinctly show that the interaction between these low-velocity pockets and the overriding longitudinal vortices is strongly altered, the latter being only slightly disturbed by the spanwise Stokes layer which develops due to the wall movement. We also notice that the ejection phenomena of low-speed fluid from the viscous sublayer to higher regions of the boundary layer and the sweeping motions of high-speed fluid towards the wall are both significantly weakened. During the first instants of the wall oscillation, the nearwall turbulent structures are already remarkably affected after one oscillating cycle. The drag-reducing flow pattern downstream of the moving wall is also described.
\end{abstract}

PACS numbers: 47.27.Lx, 47.27.Rc

\footnotetext{
${ }^{1}$ Present address: Department of Mathematics, Imperial College, 180 Queen's Gate, London SW7 2BZ, UK. URL: http://www.ma.ic.ac.uk/ ${ }^{\sim}$ par/
} 


\section{Contents}

1 Introduction 2

2 Facility and experimental techniques 3

2.1 Water channel and oscillating wall . . . . . . . . . . . . . . . . . 3

2.2 Flow visualization setup and procedures . . . . . . . . . . . . . . 4

3 Results and discussion $\quad 6$

3.1 The mean streamwise velocity and the Reynolds stresses . . . . . . . . . . . . 6

3.2 A note on the spatial transient from the foremost edge of the oscillating wall . . 7

3.3 Overhead views . . . . . . . . . . . . . . . . . . . 7

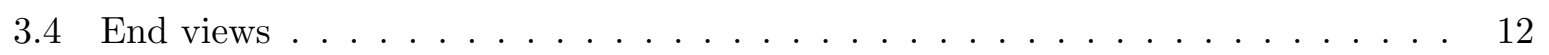

4 Summary and concluding remarks $\quad 16$

$\begin{array}{ll}\text { Acknowledgments } & 17\end{array}$

$\begin{array}{ll}\text { References } & 17\end{array}$

\section{Introduction}

Recently, great effort in the turbulent flow research community has been directed to studies on wall turbulence management, i.e. to techniques apt to manipulate turbulent structures, especially at the very proximity of the wall. Such methods aim at improving the performances of thermofluids systems, by enhancing turbulent mixing and heat transfer or by reducing noise and friction drag.

One of the simplest and most promising techniques which guarantees a reduction in wallshear stress is a spanwise sinusoidal motion of the wall. Researchers are in agreement on the fact that the turbulence activity can be attenuated by dint of oscillations of appropriate amplitude and frequency, thus yielding values of drag reduction as high as 40\% [1]-[6]. Since the pioneering work on the subject by Jung et al [1], the transversal movement of the wall has been supposed to efficiently tame the momentum transport in the proximity of the wall, where the occurrence and strength of turbulent bursts dramatically reduced during the oscillation. Akhavan et al [7] first put forward the idea of a disruption of the coherent interactions between the quasi-streamwise vortices and the underlying low-speed streaks, so that these structures now move relative to each other in the spanwise direction. They further proposed an explanation for the existence of an optimal period of oscillation for a fixed maximum wall velocity, based on the observation of the near-wall coherent structures. Soon afterwards, the first experimental study on the subject was published by Laadhari et al [2], who confirmed the view of the alteration of the near-wall turbulent regenerative cycle.

Choi et al [8] pointed out that the reduction of the mean streamwise velocity gradient at the wall is caused by a net spanwise vorticity originated by the interaction of the turbulent boundary layer with the Stokes layer which develops as a result of viscous diffusion from the surface. The importance of the shearing action of the spanwise Stokes layer on the near-wall turbulent motions was later confirmed and extended by Quadrio and Sibilla [4] and by Il-Choi et al [9]. They remarked that the low-velocity streaks need to be well merged in the region where tangential stresses are paramount in order to efficiently achieve a drag reduction effect. This vision has been recently bolstered by the numerical visualization by Quadrio and Ricco 
[6], who reported that during the very first instants of the oscillation, the wall-shear stress remains constant since the low-speed streaks are not fully embedded in the spanwise-shear region and are thus still largely unaffected. Iuso et al [10] recently presented a statistical analysis of the modification of near-wall streaks brought about by spanwise wall oscillations, based on data acquired by the particle image velocimetry technique. They found that the streaks' strength is reduced and the average velocity of these structures increased for the perturbed flow.

We herein attempt to further the understanding of the changes induced by the sinusoidal wall motion on the near-wall turbulent regenerative cycle. The study was motivated by the fact that a thorough visual experimental analysis of the flow has not been presented and that the unique flow features by which drag reduction occurs still constitute a debated topic. We thus employ flow visualizations conducted by means of the hydrogen-bubble technique to gain a vivid picture of the modified flow. The flow was observed both from above, which enables us to follow the longitudinal evolution of the low-speed streaks in the viscous sublayer, and by effective end views performed by simultaneously injecting bubbles at two vertical heights to analyse the influence of the oscillation on the interaction between the streaks, located primarily in the viscous sublayer, and the quasi-streamwise vortices, mainly present in the buffer layer and in the lower part of the logarithmic-law region [11, 12]. We also set forth the initial response of the near-wall flow to the wall motion and the drag-reducing mechanisms at work downstream of the oscillating section.

Flow visualizations conducted at the highest values of maximum wall velocity are likely to describe the spatial adjustment of the turbulent flow to the new equilibrium periodic state. Indeed, due to the limited extension of the oscillating wall section, the flow might not have fully reached the new regime which shows a statistically steady-state character, with stationary time-averaged quantities. The issue of the influence of the spatial transient from the beginning of the oscillating wall is addressed in section 3.2.

\section{Facility and experimental techniques}

The experiments and analysis were performed at the Turbulence and Turbine Cooling Research Laboratory in the Mechanical Engineering Department at The University of Texas at Austin. The laboratory equipment consisted of a low-speed recirculating water channel and a hydrogenbubble-generating device.

\subsection{Water channel and oscillating wall}

The flow visualization tests were conducted in a closed-loop channel (figure 1) where water achieved a free-stream velocity of nominally $U_{\infty}=0.18 \mathrm{~m} \mathrm{~s}^{-1}$ with one pump. The channel was free-surfaced and had a test section of $5 \mathrm{~m}$ long, $0.5 \mathrm{~m}$ wide and $0.3 \mathrm{~m}$ deep. It was made of 12.7-mm-thick cast acrylic sheets. Water flowed from a stilling tank on a false floor, where the oscillating wall mechanism was located at a streamwise position of $3.5 \mathrm{~m}$ from the inlet of the test section. The boundary layer thickness $\delta$ measured about $60 \mathrm{~mm}$, and the Reynolds number based on the momentum thickness $\theta$ and on $U_{\infty}$ was $R e_{\theta}=1400$. A 3-mm-diameter rod was located at a streamwise position of $0.5 \mathrm{~m}$ to trip the boundary layer flow. The water level for the experiments was $0.2 \mathrm{~m}$ from the false floor surface.

The oscillating section was $660 \mathrm{~mm}$ long and $450 \mathrm{~mm}$ wide and was mounted on a pair of linear bearings. The motion was given by a crank-slider mechanism connected to a Compumotor M106-178 stepping motor regulated by an IBM PC-AT computer. The spanwise 
Modification of near-wall turbulence due to spanwise wall oscillations

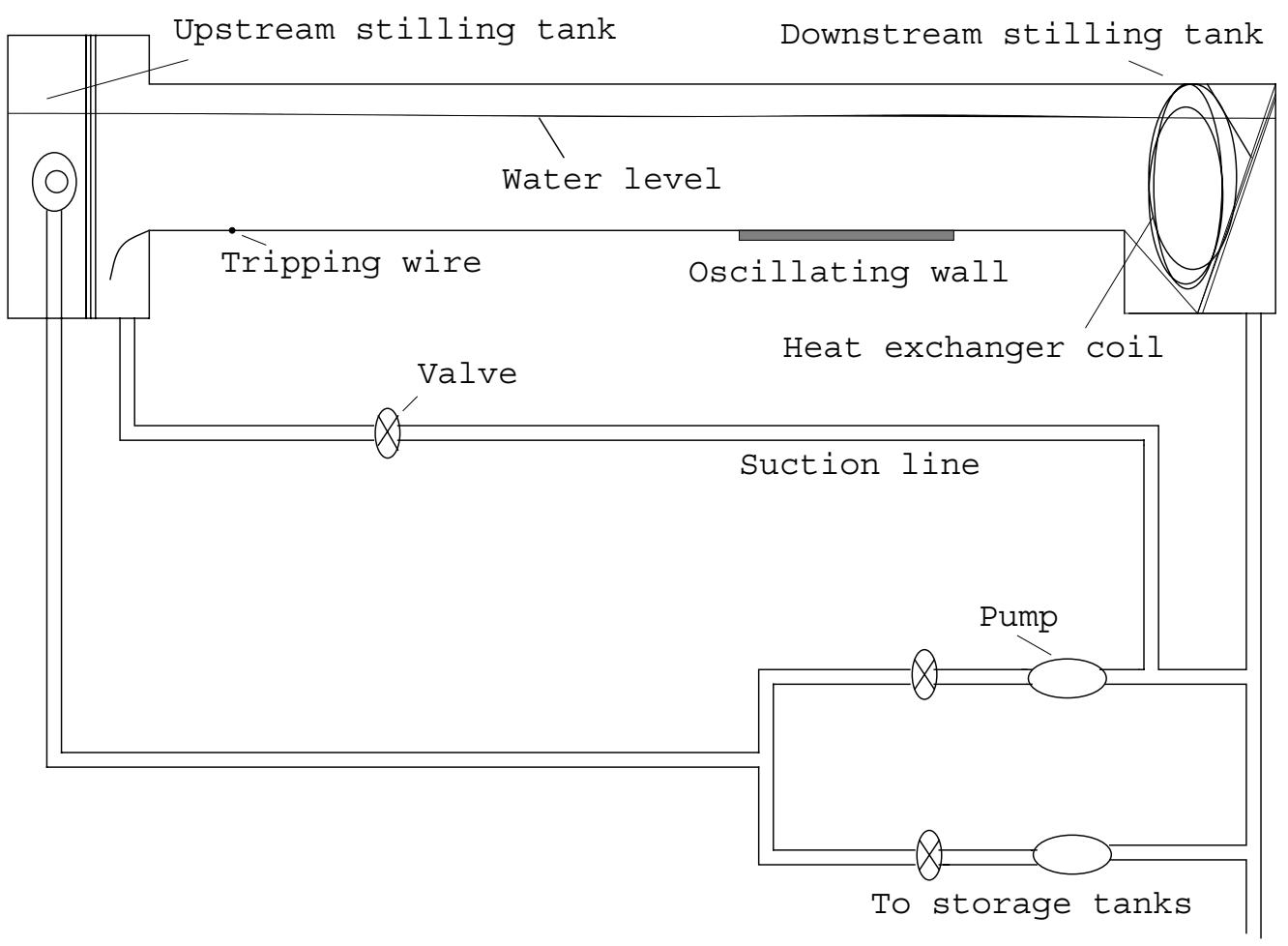

Figure 1. Schematic of water channel facility.

Table 1. Flow conditions for the stationary-wall case.

\begin{tabular}{llllll}
\hline$U_{\infty}\left(\mathrm{m} \mathrm{s}^{-1}\right)$ & $\delta(\mathrm{mm})$ & $\theta(\mathrm{mm})$ & $R e_{\theta}$ & $u_{\tau}\left(\mathrm{m} \mathrm{s}^{-1}\right)$ & $\nu / u_{\tau}(\mathrm{mm})$ \\
\hline 0.18 & 60 & 7.8 & 1400 & 0.0083 & 0.1217 \\
\hline
\end{tabular}

wall displacement $D$ followed a sinusoidal function of time:

$$
D=\frac{D_{\mathrm{m}}}{2} \sin \left(\frac{2 \pi t}{T}\right),
$$

with an estimated 3\% deviation from the ideal curve. Water was filtered to a submicron level through a two-stage process for all the experiments. A more detailed description of the water channel facility can be found in [13].

Table 1 presents the flow conditions for the stationary-wall case. They were the same as in the study by Ricco and $\mathrm{Wu}$ [14], conducted on the same experimental facility and at the same Reynolds number. The value of the friction velocity is $u_{\tau}=\sqrt{\tau_{w} / \rho}=0.0083 \mathrm{~m} \mathrm{~s}^{-1}$, where $\tau_{w}$ is the streamwise wall-shear stress for the stationary-wall case. This value is only $2 \%$ different from $u_{\tau}=0.008137 \mathrm{~m} \mathrm{~s}^{-1}$ given by the following correlation [15] for the skin-friction coefficient $C_{f}$ :

$$
C_{f}=2 \tau_{w} /\left(\rho U_{\infty}^{2}\right)=0.025 R e_{\theta}^{-0.25},
$$

where $\rho$ is the density of water.

\subsection{Flow visualization setup and procedures}

The boundary layer flow was visualized by hydrogen bubbles generated by hydrolysis of water, obtained by means of a platinum wire continuously energized as the cathode in an electric 


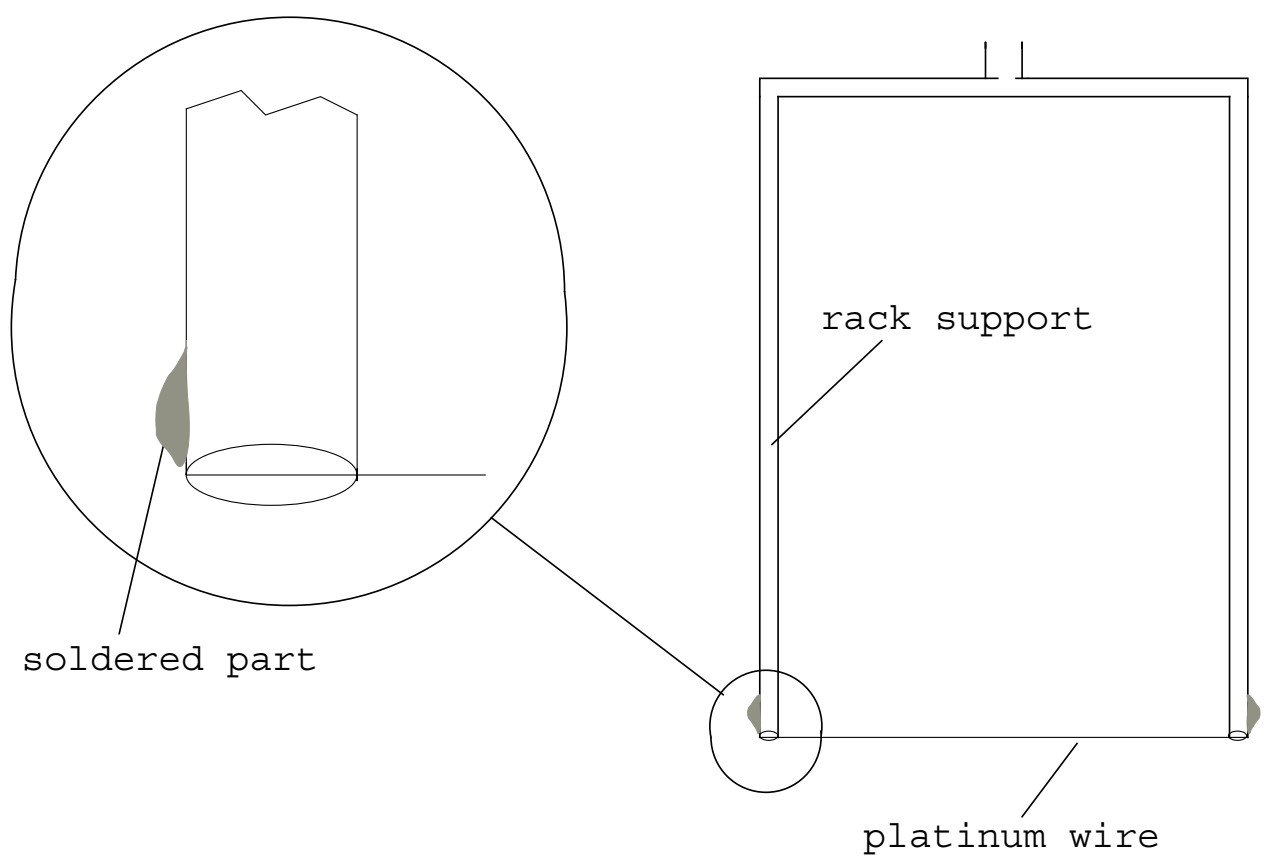

Figure 2. Platinum wire support.

circuit [16]. The wire had a length of $250 \mathrm{~mm}$ and a diameter of $0.025 \mathrm{~mm}\left(\sim 0.2 \nu / u_{\tau}\right)$ so that even at the maximum velocity of $0.18 \mathrm{~m} \mathrm{~s}^{-1}$, no von Kármán vortex street was shed. Such a thin wire generated bubbles of the order of half the wire diameter, which allowed us to consider the buoyancy effects negligible in the region of visualization [17]. A fork-like support rack of a $4 \mathrm{~mm}$ diameter circular brass rod served both the purpose of conductor for the bubble-wire circuit and of tensioning agent to hold the bubble-wire taut. The support was inserted from the free surface and oriented parallel to the floor and perpendicular to the streamwise direction. For the simultaneous visualization of turbulent structures at different heights, two wires were placed parallel to each other and again perpendicular to the mean flow. An illustration of the platinum wire support is shown in figure 2. A limited quantity of sodium sulphate $\left(\sim 0.15 \mathrm{~g} \mathrm{Na}_{2} \mathrm{SO}_{3} \mathrm{I}^{-1}\right.$ of water) was added to the water to favour the bubble generation and to produce denser bubble lines for a better visual contrast between the bubbles and the dark background.

A TRV65 Sony model video camera (connected to a Toshiba VHS tape unit) with a typical frame velocity of 24 frames $\mathrm{s}^{-1}$ was employed for image recording. The image post-processing was performed on a Macintosh computer with the NIH Image program, written by the US National Institutes of Health and available on the Internet without charge. For overheadview experiments, two halogen spotlights continuously illuminated the bubble sheet from the sides of the channel and the camera was positioned on top of the channel, looking downward perpendicularly to the oscillating surface. Furthermore, a glass window was placed on the free surface of the water below the video camera to avoid disturbances on the images due to the movement of the water surface during the oscillation. The video camera was located at the downstream end of the channel, behind the second stilling tank for end views. The heat exchanger coil, the eddy-damping honeycomb and the contraction planes were removed to obtain visual access to the test section. A 3-mm-thick $\left(\sim 25 \nu / u_{\tau}\right)$ laser sheet generated from a laser beam by a semicylindrical lens provided the continuous illumination. The plane was oriented parallel to the spanwise direction and perpendicular to the mean flow direction and to the wall. Once the bubble sheet was generated, it was distorted by the turbulence and then lit by the laser plane. 
Modification of near-wall turbulence due to spanwise wall oscillations

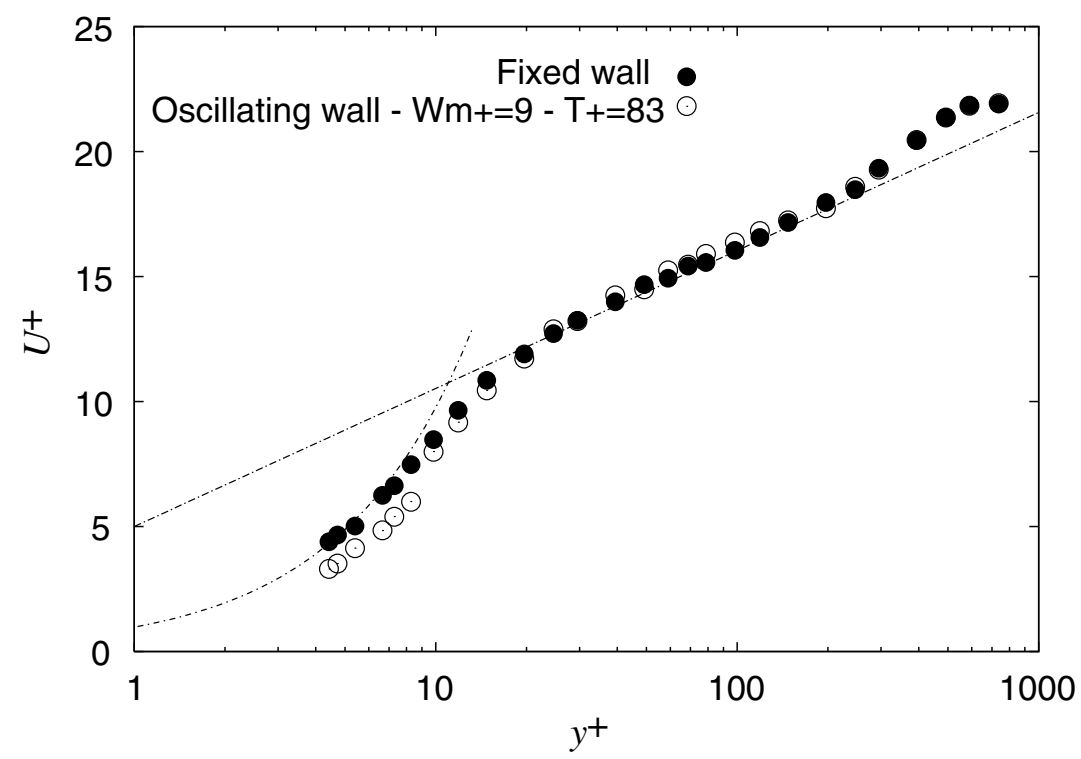

Figure 3. Mean velocity profiles of stationary- and oscillating-wall case $\left(W_{\mathrm{m}}^{+}=\right.$ 9 and $T^{+}=83$ ) scaled with the friction velocity of the stationary-wall case. The near-wall trend is $U^{+}=y^{+}$and straight line is $U^{+}=(1 / \kappa) \ln y^{+}+C$, where $\kappa=0.41$ and $C=5.0[20]$.

For the analysis of the overhead-view images, a definition of low-speed streak was adopted following the works by Oldaker and Tiederman [18] and by Tiederman et al [19]. A low-speed streak was recognized as a clearly identifiable single longitudinal structure of bubbles with streamwise length at least three times the apparent average streaks' spacing. No constraint of identification was placed upon the minimum spanwise spacing between two low-speed streaks. For each oscillating condition, four observers analysed an average number of 25 independent images and identified a minimum of 100 to a maximum of 400 streaks.

\section{Results and discussion}

\subsection{The mean streamwise velocity and the Reynolds stresses}

The mean streamwise velocity and Reynolds stress profiles for the modified flow of the study by Ricco and $\mathrm{Wu}[14]$ are introduced in this section to briefly illustrate the effects of the wall oscillation on the velocity field (figures 3 and 4 ). The oscillatory parameters ${ }^{2}$ are set to $W_{\mathrm{m}}^{+}=9$ and $T^{+}=83$, where the + sign indicates non-dimensionalization with inner variables of the stationary-wall case, namely with the friction velocity $u_{\tau}$ and with the kinematic viscosity $\nu$ of the fluid. The effect of the oscillation on the mean velocity profile extends up to $y^{+} \approx 20$ and, for these oscillatory parameters, a $25 \%$ reduction of wall friction is calculated. The influence on the turbulence shear stresses $-\overline{u v}$ is more marked than on the mean velocity so that the profiles are reduced up to $y^{+} \approx 200$. The maximum value decreases by $\sim 25 \%$ when the oscillations are imposed.

${ }^{2} W_{\mathrm{m}}^{+}$is the maximum wall velocity and is related to the other oscillation parameters by the following: $W_{\mathrm{m}}^{+}=\pi D_{\mathrm{m}}^{+} / T^{+}$. 


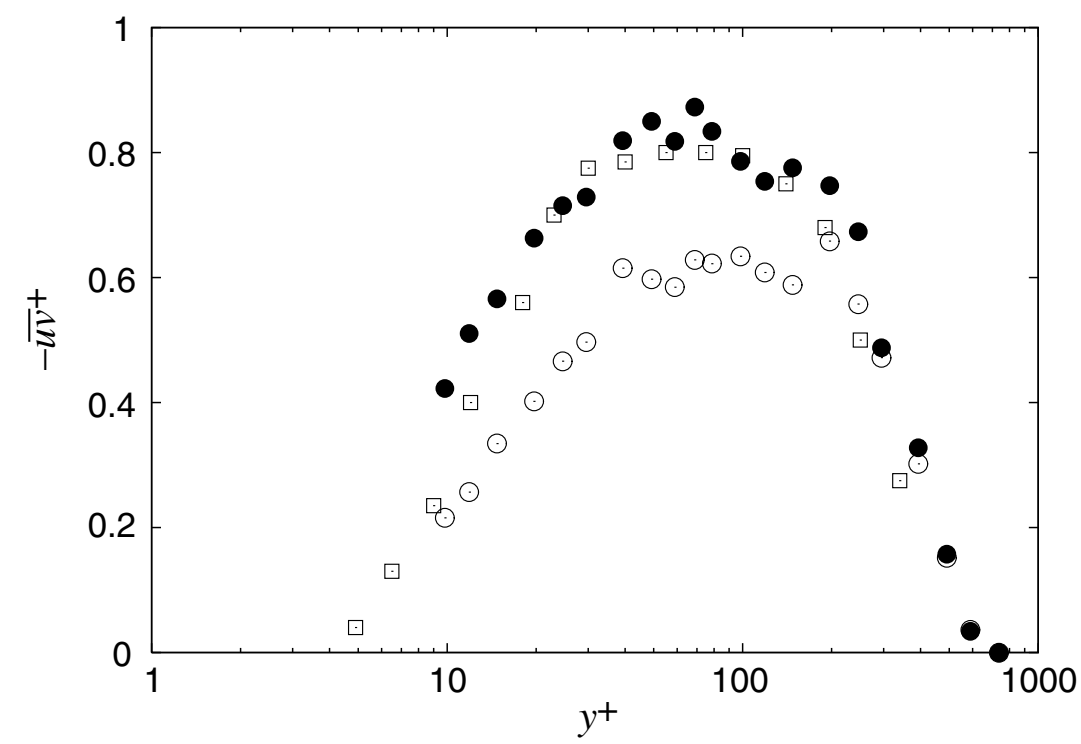

Figure 4. Reynolds stress profiles of stationary- and oscillating-wall case $\left(W_{\mathrm{m}}^{+}=9\right.$ and $\left.T^{+}=83\right)$ scaled with the friction velocity of the stationary-wall case. White squares display data of DeGraaff and Eaton [21] for a turbulent boundary layer with $R e_{\theta}=1430$.

\subsection{A note on the spatial transient from the foremost edge of the oscillating wall}

According to the recent experimental work by Ricco and $\mathrm{Wu}$ [14] conducted on the same facility of the present study, the spatial evolution of the modified flow elapses at a downstream location of $x \approx 4 \delta\left(x^{+} \approx 3000\right)$, for $W_{\mathrm{m}}^{+}=11.3$ and $T^{+}=67$. Further, by converting the temporal transient obtained via DNS into the spatial transient through the concept of the convection velocity, Quadrio and Ricco [6] found that the transient strongly depends on $W_{\mathrm{m}}^{+}$, so that doubling $W_{\mathrm{m}}^{+}$ could double the length of the transient evolution, whereas the period of oscillation $T^{+}$has no significant effect as long as $50<T^{+}<200$.

In view of the above results and since the flow visualization analysis is conducted by positioning the wire at $x=6.4 \delta\left(x^{+} \approx 4000\right)$ from the foremost edge of the moving section, it is likely that cases 6-9 in table 2 , hence the ones with $W_{\mathrm{m}}^{+}>11.3$, describe the flow while still adjusting to the new state, whereas the cases with lower values of $W_{\mathrm{m}}^{+}$illustrate the new fully developed regime. The wire was not positioned farther downstream lest the flow beyond the rearmost edge of the plate might influence the upstream flow. In all the figures, these four cases are the ones with values of drag reduction higher than $30 \%$. Considering that a complete study on the spatial transient is still missing, a detailed analysis of the dependence of the spatial evolution on the oscillatory parameters is of primary interest to attain a sound understanding of wall-bounded turbulence modified by spanwise wall oscillations.

\subsection{Overhead views}

For this set of experiments, the wire was held taut at $x=6.4 \delta\left(x^{+} \approx 4000\right)$, from the beginning of the plate at $y^{+}=5(y \approx 0.5 \mathrm{~mm})$. The flow organizes in the well-known streaky pattern for the stationary-wall case [22], as shown in the left image of figure 5 and in the animation of figure 6. Consistently with numerous previous investigations (see, amongst these studies, $[23,24])$, the streaks' spacing $\lambda^{+}$is about 100 non-dimensional viscous units, as shown in table 2 . 
Table 2. Streaks' spacing, length, angle of inclination with respect to streamwise direction and drag reduction values (from DNS study by Quadrio and Ricco [26]) for different oscillatory conditions. Case 3 is the oscillating-wall case of figure 5 and case 4 is the oscillating case of animation of figure 7 . The last column on the right indicates whether the modified flow has reached the new fully developed state $(\mathrm{FD})$ or is still in its spatial transient (ST).

\begin{tabular}{llrllllllll}
\hline Case & $T^{+}$ & $D_{\mathrm{m}}^{+}$ & $W_{\mathrm{m}}^{+}$ & $\lambda^{+}$ & $\lambda_{\text {oscill }}^{+}$ & $l^{+}$ & $l_{\text {oscill }}^{+}$ & $\gamma(\mathrm{deg})$ & DR (\%) & Regime \\
\hline 0 & $\infty$ & 0 & 0 & 103.9 & - & 435.3 & - & 0 & 0 & - \\
1 & 208.4 & 240 & 3.6 & 103.7 & 98.4 & 406 & 385.2 & 18.8 & 10 & FD \\
2 & 208.4 & 360 & 5.4 & 110.3 & 102 & 388.6 & 359.3 & 24 & 14.5 & FD \\
3 & 167 & 480 & 9 & 118.4 & 102 & 341.2 & 295.5 & 27.1 & 25 & FD \\
4 & 120 & 360 & 9.4 & 127.9 & 108.5 & 350.9 & 297.8 & 27.1 & 28 & FD \\
5 & 138.8 & 480 & 10.9 & 140.8 & 117.9 & 331.8 & 277.6 & 27.9 & 30 & FD \\
6 & 83.3 & 360 & 13.6 & 142.8 & 116.9 & 306.2 & 250.6 & 29.8 & 33 & ST \\
7 & 92.6 & 480 & 16.3 & 178.8 & 141.3 & 266.2 & 210.4 & 30.1 & 37.5 & ST \\
8 & 67.5 & 480 & 22.3 & 180.5 & 142.2 & 238.3 & 187.6 & 33.5 & 38 & ST \\
9 & 75.7 & 480 & 19.9 & 191.8 & 149.8 & 261.9 & 204.5 & 35.5 & 39 & ST \\
\hline
\end{tabular}
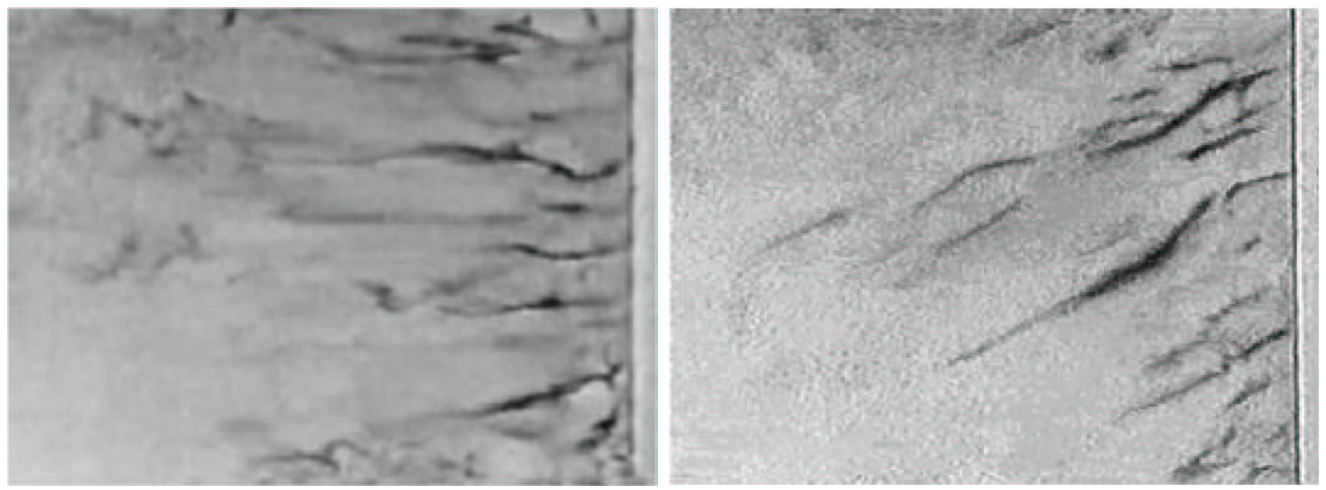

Figure 5. Overhead views with the platinum wire positioned at $y^{+}=5$. Range of view was $750 \nu / u_{\tau}$ spanwise and $1000 \nu / u_{\tau}$ streamwise; flow direction is from right to left. Left image: stationary-wall case; right image: oscillating-wall case $\left(T^{+}=167, D_{\mathrm{m}}^{+}=480\right.$ and $\left.W_{\mathrm{m}}^{+}=9\right)$ at maximum tilting of near-wall structures $\gamma=27^{\circ}$ (phase of wall oscillation $\phi \approx T^{+} / 2$ ).

The elongated structures stretch along the streamwise direction as soon as the bubbles detach from the wire and then show the characteristic meandering motion at a distance of about 50 wall units from the wire. Eventually, the streaks lose their characteristic structure when they burst upward.

When the wall oscillates, the streaky structure can still be identified, but it is more homogeneous, shorter in length and convects downstream for a shorter time. The structures deviate from the streamwise direction by periodically inclining themselves, as visible in the right image of figure $5\left(T^{+}=167, D_{\mathrm{m}}^{+}=480\right.$ and $\left.W_{\mathrm{m}}^{+}=9\right)$ and in the animation of figure $7\left(T^{+}=120\right.$, $D_{\mathrm{m}}^{+}=360$ and $\left.W_{\mathrm{m}}^{+}=9.4\right)$. The streaks' orientation is brought about by the fact that while the upstream part of a streak keeps moving laterally, being close to the wire and to the wall, the downstream part only convects downstream. Indeed, this part rises to a height at which it is not influenced by the spanwise shearing motion and it advects freely. Furthermore, for the case 
Modification of near-wall turbulence due to spanwise wall oscillations

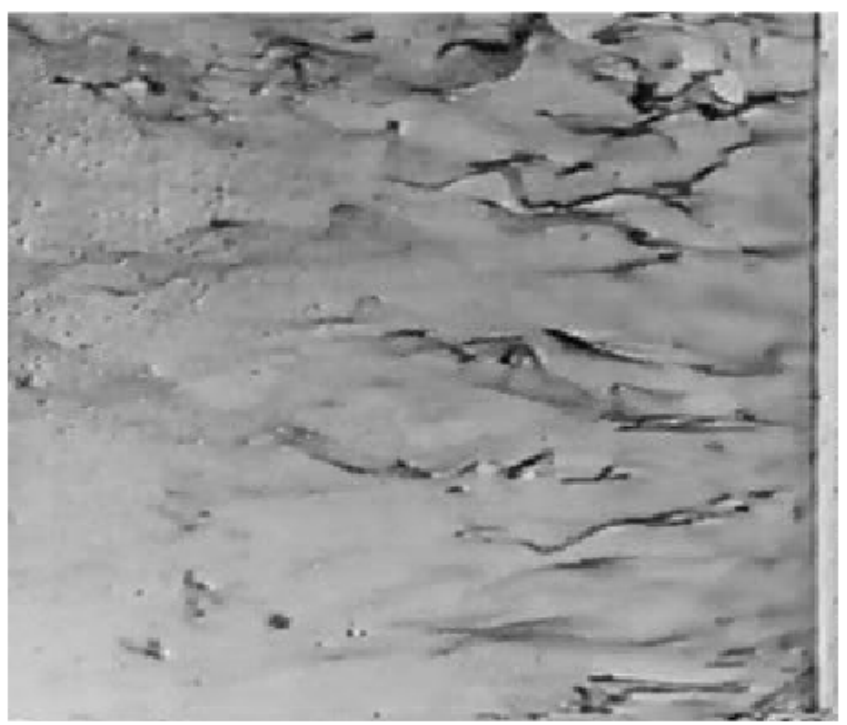

Figure 6. Top-view animation for stationary-wall case with the platinum wire positioned at $y^{+}=5$. Range of view was $1100 \nu / u_{\tau}$ spanwise and $1450 \nu / u_{\tau}$ streamwise. The total time of the animation is $t^{+}=180$.

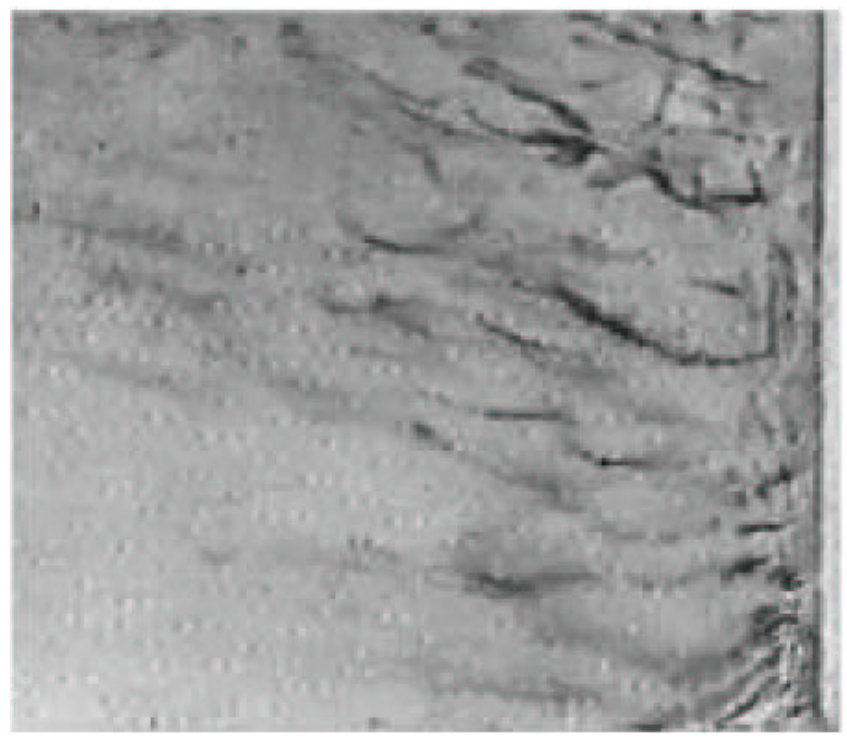

Figure 7. Top-view animation for oscillating-wall case $\left(T^{+}=120, D_{\mathrm{m}}^{+}=360\right.$ and $W_{\mathrm{m}}^{+}=9.4$ ) with the platinum wire positioned at $y^{+}=5$. Range of view is $1100 \nu / u_{\tau}$ spanwise and $1450 \nu / u_{\tau}$ streamwise. The total time of animation is $t^{+}=180$.

of the animation of figure 7 , the streaky structures incline themselves to the maximum angle with respect to the streamwise direction when the wall underneath is about to stop and when the spanwise velocity of the Stokes layer at the height at which the bubbles detach from the wire is at its maximum. During the oscillating cycle, the structures direct themselves along the streamwise direction when the wall reaches its maximum velocity and the spanwise velocity of the Stokes layer at the height of the bubble wire is null. 


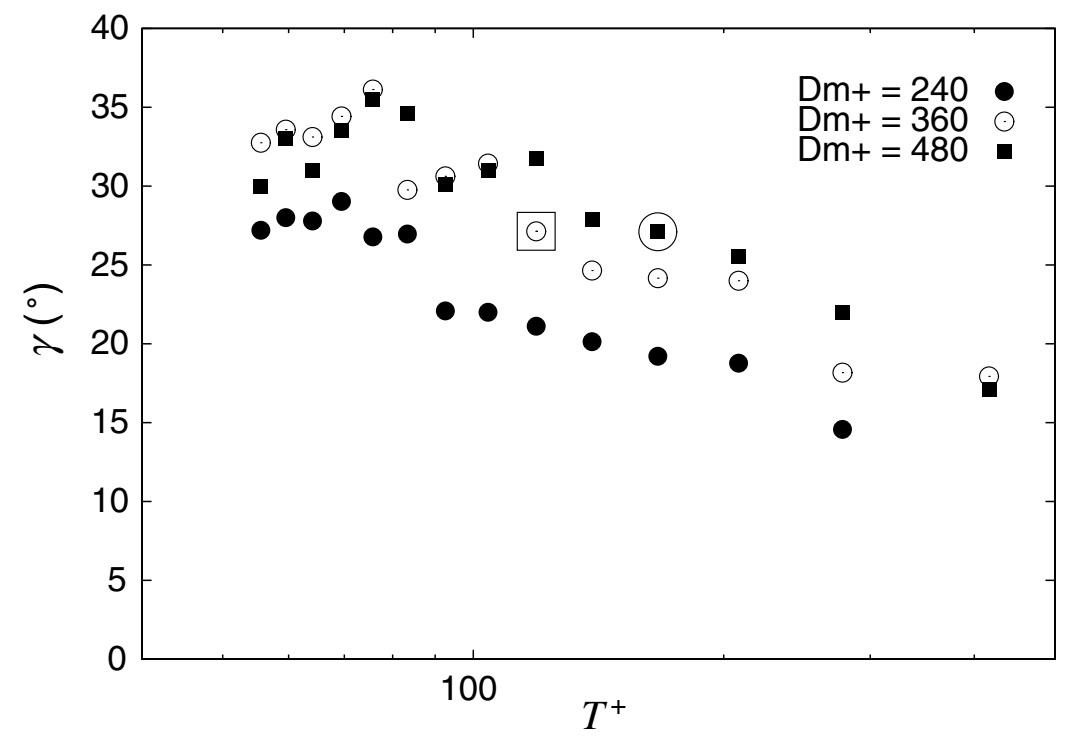

Figure 8. Maximum angle of streaks' inclination $\gamma$ with respect to the streamwise direction as a function of $T^{+}$. In this figure and in the following ones, the circled symbol indicates the oscillating-wall case depicted in the right-hand-side image of figure $5\left(T^{+}=167, D_{\mathrm{m}}^{+}=480\right.$ and $W_{\mathrm{m}}^{+}=9$, case 3 in table 2$)$ and the squaredsymbol indicates the oscillating-wall case in the animation of figure $7\left(T^{+}=120\right.$, $D_{\mathrm{m}}^{+}=360$ and $W_{\mathrm{m}}^{+}=9.4$, case 4 in table 2 ).

To study the variation of the maximum streak angle $\gamma$ of inclination with respect to the parameters of the oscillation, the period was varied between $T^{+}=56$ and 415 and the peak-topeak amplitudes were $D_{\mathrm{m}}^{+}=240,360$ and 480 . The angle $\gamma$ was measured when a streak reached the maximum inclination from the mean flow direction. The maximum angle of inclination of about $30^{\circ}$ detected in our visualizations compares well with an estimate given by the vector of the convection velocity $U_{\mathrm{c}}$ at this height $\left(U_{\mathrm{c}}^{+} \approx 10\right.$, as shown by Krogstad et al [25]) and the vector of the peak spanwise velocity of the Stokes layer, also at $y^{+}=5$ and for $T^{+}=67$ and $W_{\mathrm{m}}^{+}=17$. The maximum angle can then be estimated as follows:

$$
\gamma\left(y^{+}=5 ; T^{+}=67 ; W_{\mathrm{m}}^{+}=17\right)=\arctan \left(\frac{W_{\mathrm{m}}^{+} \exp \left(-y^{+} \sqrt{\pi / T^{+}}\right)}{U_{\mathrm{c}}^{+}}\right) \approx 30^{\circ} .
$$

As mentioned in section 3.2 , because of the high value of the maximum wall velocity $W_{\mathrm{m}}^{+}=17$, this case probably describes the spatial transient, so that an even higher maximum angle of inclination might be achieved with a longer oscillating wall section.

Figure 8 shows $^{3}$ the trends of $\gamma$ as a function of $T^{+}$for the three amplitudes and figure 9 presents the trend of $\gamma$ as a function of the amount of drag reduction for the cases of table $2 .{ }^{4} \mathrm{We}$

\footnotetext{
${ }^{3}$ Data in figure 8 which might describe the spatial transient are the ones with $W_{\mathrm{m}}^{+}>11$, namely the ones with $T^{+}<68$ for $D_{\mathrm{m}}^{+}=240$, with $T^{+}<103$ for $D_{\mathrm{m}}^{+}=360$ and with $T^{+}<137$ for $D_{\mathrm{m}}^{+}=480$.

${ }^{4}$ The values of skin-friction reduction are from the numerical study by Quadrio and Ricco [26] which presents the dependence of the amount of drag reduction on the oscillation parameters for $R e_{\tau}=200$. The fact that these data are employed for comparison in the present analysis despite their lower Reynolds number is justified by the results of Ricco and $\mathrm{Wu}$ [14], which show that drag reduction does not depend on this non-dimensional parameter, as long as $\operatorname{Re}_{\theta} \leq 1400$.
} 


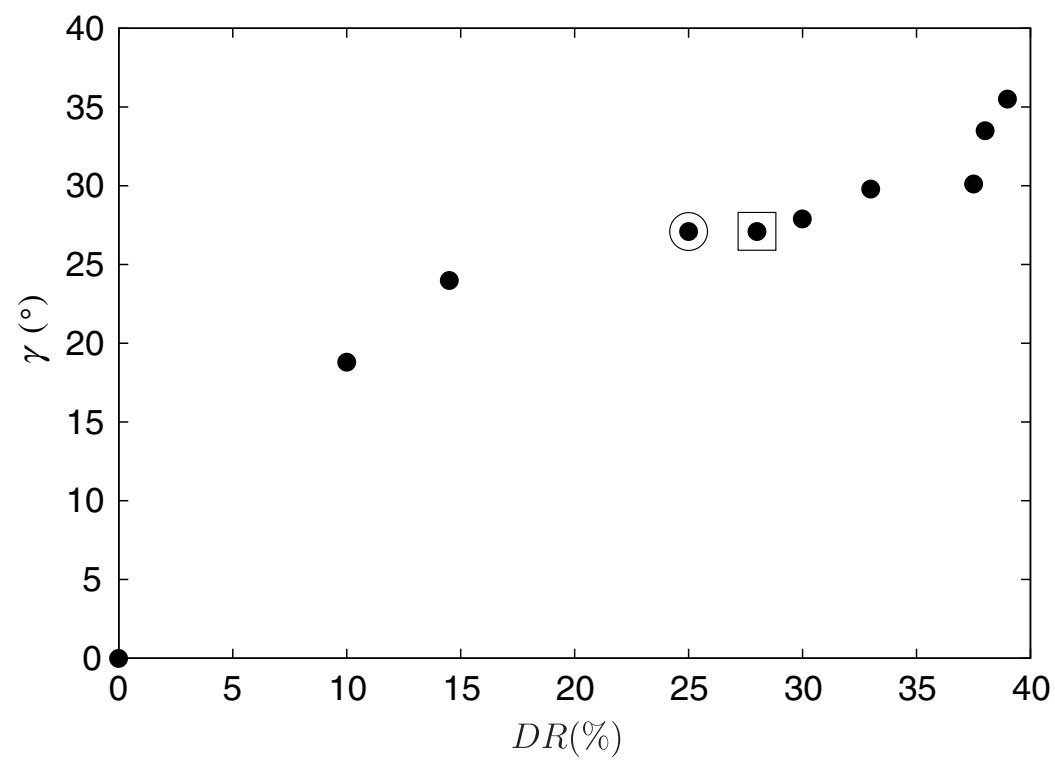

Figure 9. Angle of inclination of streaks with respect to streamwise direction as a function of drag reduction (DR).

observe that the behaviour of the angle $\gamma$ as a function of the period $T$ is similar to the trend of drag reduction $[14,26]$ and that $\gamma$ increases with increasing drag reduction. For a constant wall displacement $D_{\mathrm{m}}, \gamma$ increases as $T$ decreases until an optimum value is reached $\left(60<T^{+}<80\right)$. The amount of drag reduction also presents an optimum period for a fixed $D_{\mathrm{m}}^{+}$and it is found that this period is lower than the corresponding one for fixed $W_{\mathrm{m}}^{+}\left(T^{+} \approx 120\right)$, as verified by $[14,26]$. The tilting of the near-wall structures is accompanied by a reduction of turbulent activity as the structures appear more homogeneous and uniform when excited by the wall motion. This result is in accordance with the analogous numerical visualizations produced by Quadrio and Ricco [6], which show that the tilting character of the near-wall structures occurs simultaneously with a decrease of the turbulence level. The cyclic shift of structures was also studied via DNS by Akhavan et al [7], who observed progressively wider and weaker structures as the flow stabilizes to the new modified character.

Similar to other drag-reduction techniques, such as polymer injection $[18,19,27,28]$, the oscillating wall motion brings about an increment of the average streak spacing $\lambda_{\text {oscill }}^{+},{ }^{5}$ as seen in figure 10 and in table 2. A reduction of friction of about $40 \%$ goes together with an increment of streaks' spacing of about one-third when values are scaled with the relative inner variables, while the distance doubles in physical dimensions. The spacing trend for the oscillating-wall technique was slightly lower than the distributions given by the polymer-injection technique and it was unaffected for drag-reduction values smaller than about $25 \%$. This is probably due to the fact that the oscillating wall generates a different turbulent flow, while the injection methods guarantee a drag-reduction effect by maintaining similar turbulent features to the natural wallbounded flow. The effect of the oscillation was more remarkable on the length of the streaks, as seen in figure 11 and in table $2: l_{\text {oscill }}^{+}$was reduced by more than $50 \%$ for a drag reduction of about $40 \%$.

\footnotetext{
${ }^{5}$ A quantity is indicated by superscript + and by subscript oscill when scaled with inner variables of the relative oscillating-wall case, i.e. with $u_{\tau, o}=\sqrt{\tau_{w, o} / \rho}$, where $\tau_{w, o}$ is the mean streamwise wall-shear stress in the oscillatingwall case.
} 
Modification of near-wall turbulence due to spanwise wall oscillations

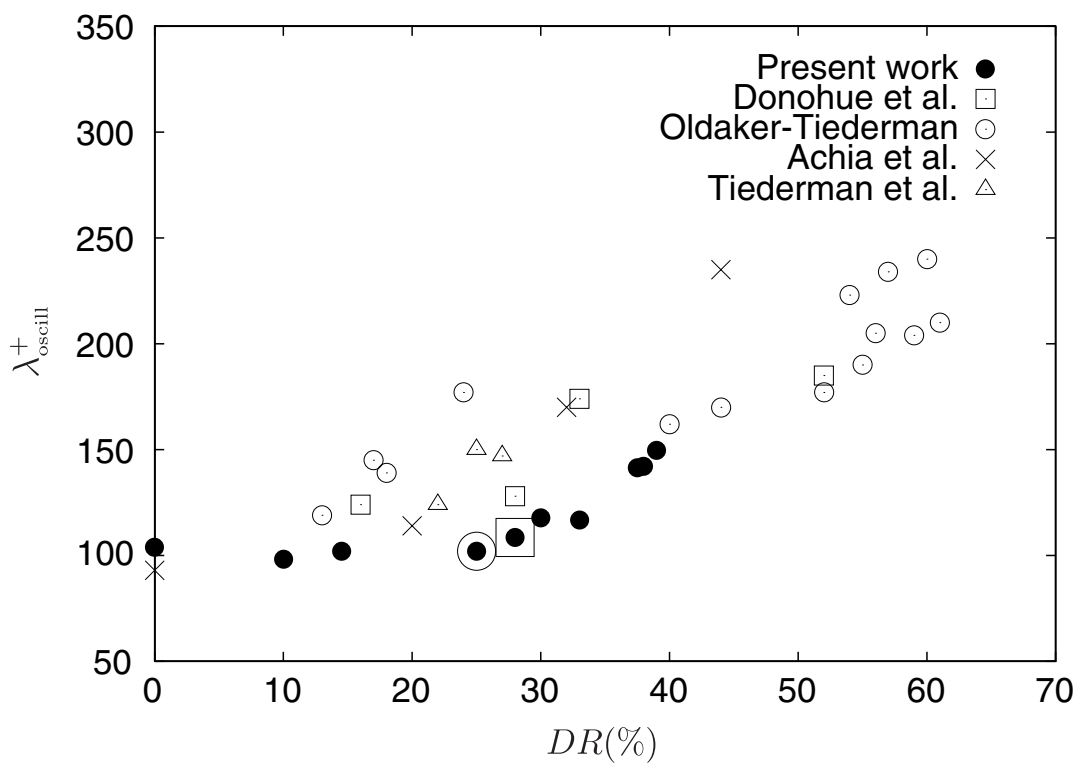

Figure 10. Streaks' spacing as a function of drag reduction.

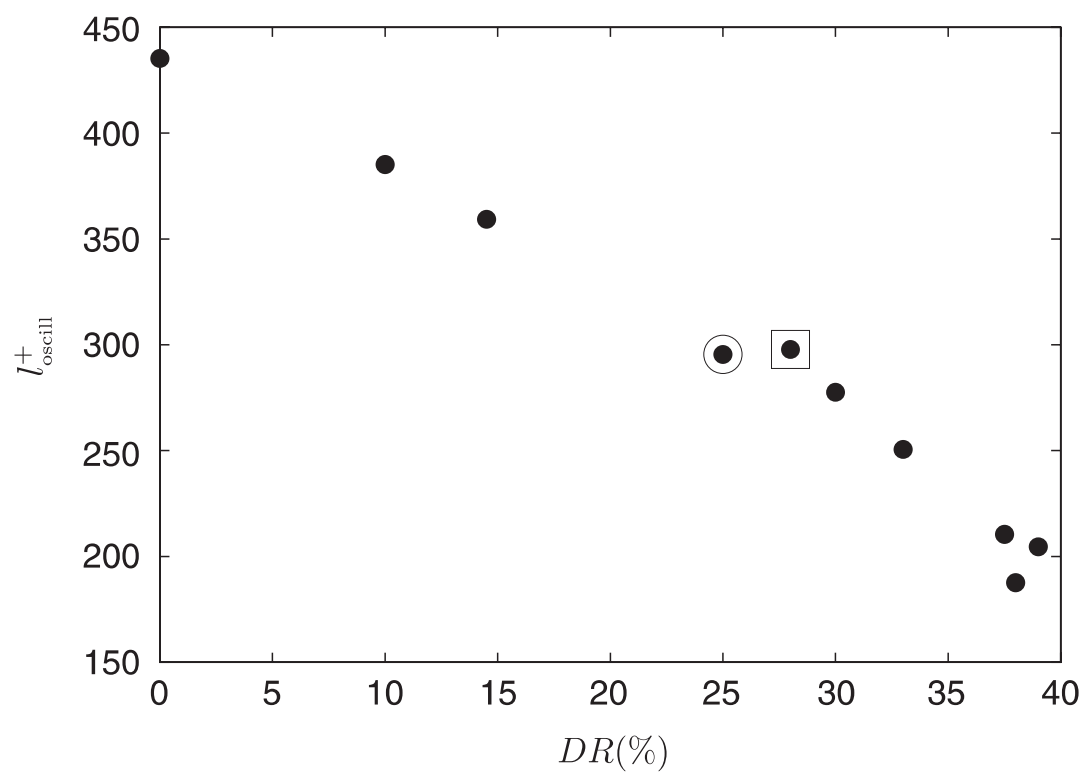

Figure 11. Streaks' length as a function of drag reduction.

\subsection{End views}

This section describes the images of the stationary-wall cases and of the oscillating-wall cases $\left(T^{+}=67, D_{\mathrm{m}}^{+}=360\right.$ and $\left.W_{\mathrm{m}}^{+}=17\right)$ from the end of the channel. For these conditions of oscillation, the visualizations are likely to illustrate the flow while still adjusting to the new modified regime, according to the discussion of section 3.2.

A first vivid impression of the effect of the oscillation on the near-wall turbulent structures can be seen in the animations of figures 12 and 13, showing one stationary-wall case and one oscillating-wall case with the platinum wire located at $y^{+}=25$ and the laser visualization plane 


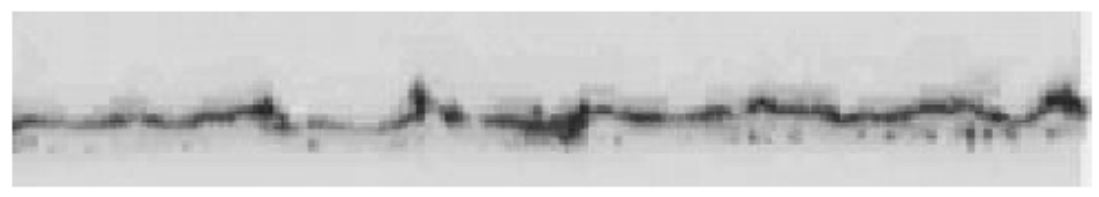

Figure 12. End-view animation for the stationary-wall case with the platinum wire positioned at $y^{+}=25$ and with the laser sheet located at a distance of $\Delta x^{+}=240$ downstream of the wire. The total time of the animation is $t^{+}=300$ and the spanwise window is $900 \nu / u_{\tau}$.

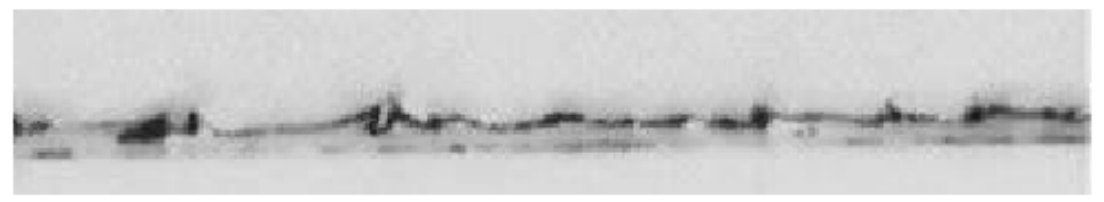

Figure 13. End-view animation for the oscillating-wall case $\left(T^{+}=67, D_{\mathrm{m}}^{+}=360\right.$ and $\left.W_{\mathrm{m}}^{+}=17\right)$ with the platinum wire positioned at $y^{+}=25$ and with the laser sheet located at a distance of $\Delta x^{+}=240$ downstream of the wire. The total time of the animation is $t^{+}=300$ and the spanwise window is $900 \nu / u_{\tau}$.

at $\Delta x^{+}=240$ downstream of the wire. The influence of the wall motion is significant and it can be quantified by a $70 \%$ reduction of lift-up motions, here defined by the bubble line reaching at least a threshold of $y^{+}=60$. For this analysis, 31 images of 900 viscous lengths in the spanwise direction were analysed for each case. The time interval between two images was about 270 viscous time units, for a total time of about 8500 time units.

Figures 14-16 present a series of images and animations of the stationary- and oscillatingwall cases. The laser plane was located at a downstream distance of $\Delta x^{+}=160$ from the wires, which were at $y^{+}=5$ and 45 . Positioning the wires at these heights and at this distance from each other allowed the visualization of both the low-speed streaks and the quasi-streamwise vortices. In the stationary-wall case, the hydrogen bubble line originating at $y^{+}=5$ shows the characteristic conglomeration of the low-speed streaks, which eventually erupt into ejection events farther from the wall and influence the vortical structures at $y^{+}=45$. The vortices are seen to lift low-speed fluid upward (bursting) and move high-speed fluid towards the wall (sweeping).

The wall oscillation produces a marked change on the streaky structure, which appears more homogeneous and sporadic, clearly indicating a reduced tendency for streaks formation. When a streak is identified, it exhibits a lateral shifting movement as it is being dragged along by the spanwise shear layer. The rise of streaks higher than $y^{+}=15$ are very rare, occurring only once every $64 t^{+}$in a window of analysis of $\Delta z^{+}=900$. The smearing action on the streaks causes the decrease of the burst-sweep activity in the buffer region because the quasi-streamwise vortices are less likely to lift low-speed fluid upward and the fewer streaks find themselves below vortices to which they were not originally dynamically paired. The near-wall transversal flow also works to impede wallward sweeping motions of high-speed fluid, which are known to be greatly responsible for high wall-shear stress [11]. The shearing action of the Stokes layer was also supported by Akhavan et al [7], who remarked that the intense spanwise velocity gradients should occur in the region $0<y^{+}<15$ for an optimal drag-reduction effect. The few streaks that can still be identified as a clear conglomeration of bubbles show an oscillatory movement in the spanwise direction. Indeed, the trace of streaks moves laterally, stops and then accelerates towards the opposite direction. This motion is distinctly depicted 
A.

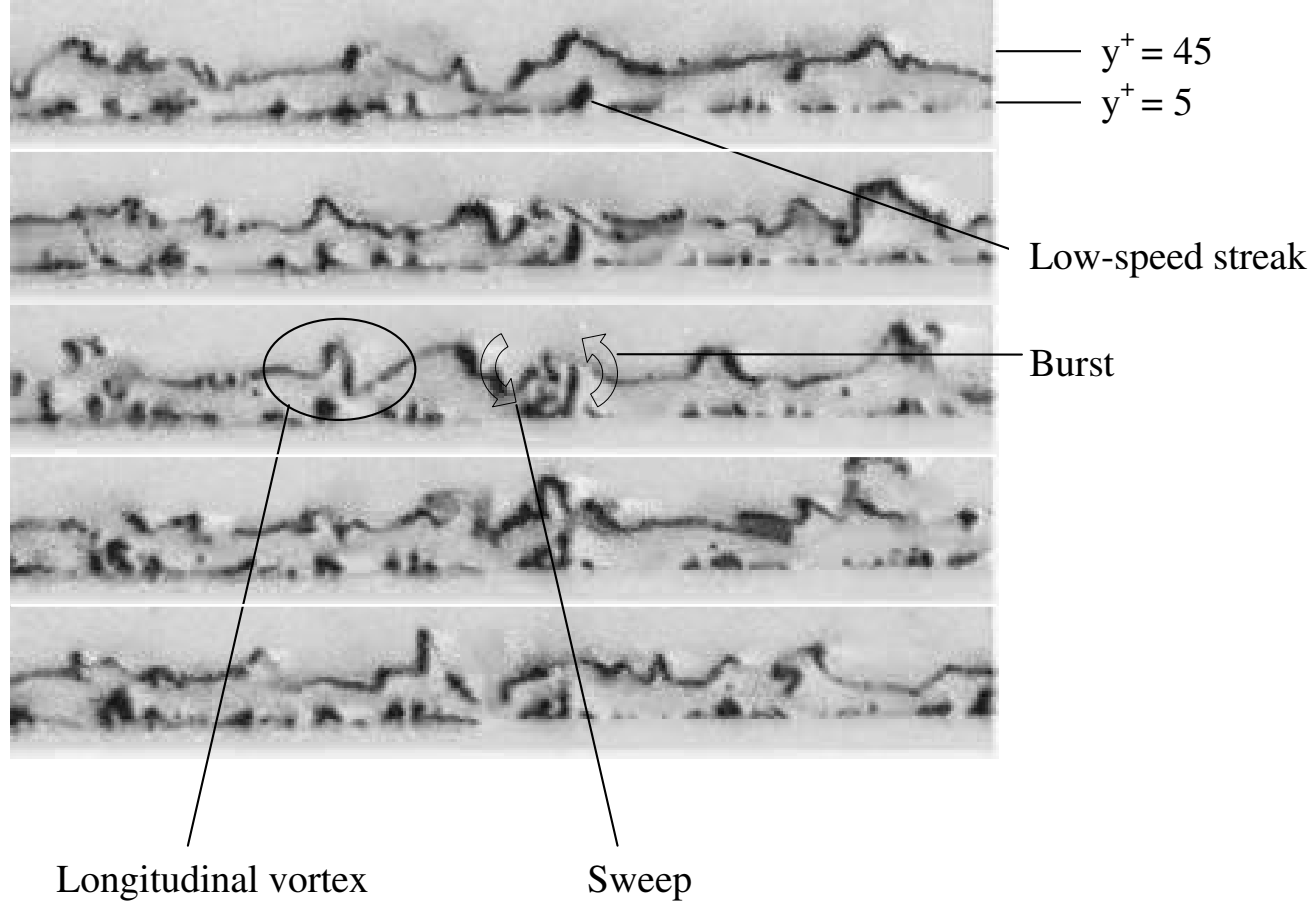

B.

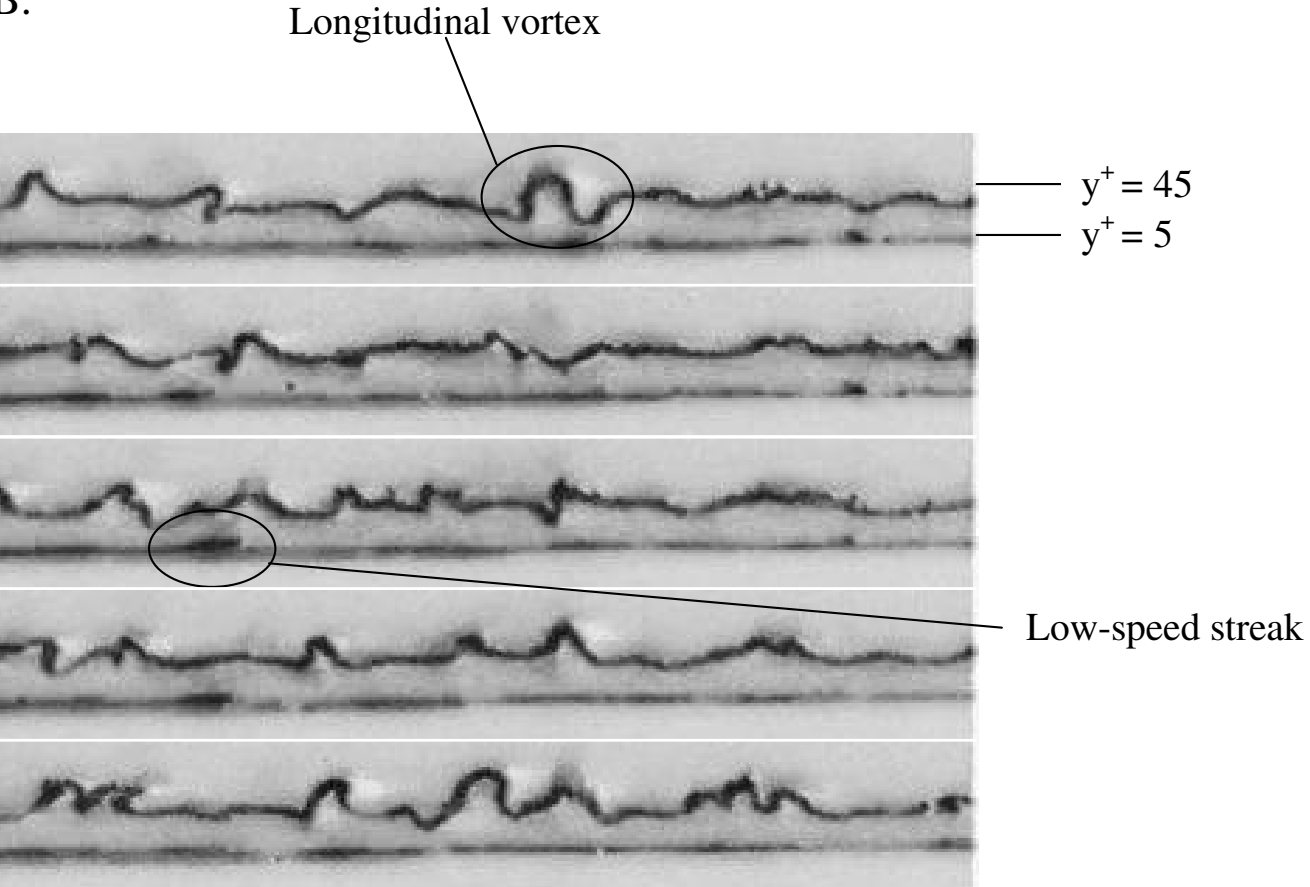

Figure 14. End views with the platinum wires at $y^{+}=5$ and 45 . The wall is either stationary (A) or moving (B) $\left(T^{+}=67, D_{\mathrm{m}}^{+}=360\right.$ and $\left.W_{\mathrm{m}}^{+}=17\right)$ and the images go from top to bottom. A time of $5.4 t^{+}$between two images is estimated and the spanwise window is $900 \nu / u_{\tau}$. 


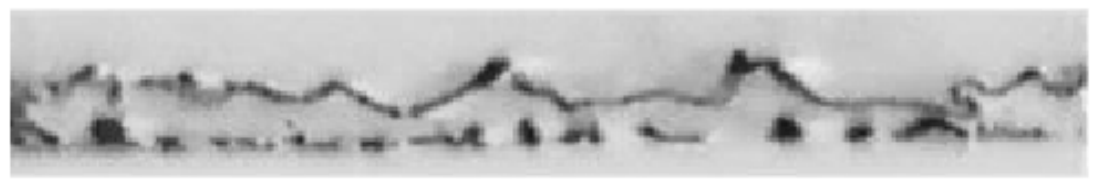

Figure 15. End-view animation for the stationary-wall case with the platinum wires positioned at $y^{+}=5$ and 45 . The total time of the animation is $t^{+}=300$ and the spanwise window is $900 \nu / u_{\tau}$.

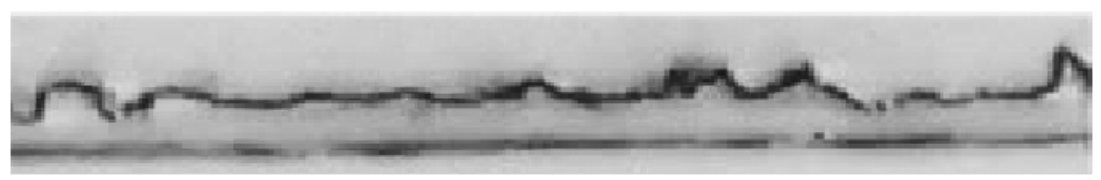

Figure 16. End-view animation for oscillating-wall case $\left(T^{+}=67, D_{\mathrm{m}}^{+}=360\right.$ and $\left.W_{\mathrm{m}}^{+}=17\right)$ with the platinum wires positioned at $y^{+}=5$ and 45 . The total time of the animation is $t^{+}=300$ and the spanwise window is $900 \nu / u_{\tau}$.

in figure 14, where the streak on the left of the images is visible for about half a cycle of oscillation.

As expected, this motion does not follow the movement of the wall since the streaky structure is at a higher location $\left(y^{+} \approx 5\right)$. By integrating the analytical solution of the second Stokes problem, the maximum lateral displacement $\Delta z_{\text {max-streak }}^{+}$of a streak can be estimated and then compared with the average movement of a streak in the images. The theoretical value is as follows:

$$
\Delta z_{\text {max-streak }}^{+}\left(y^{+}=5 ; T^{+}=67 ; W_{\mathrm{m}}^{+}=17\right)=\frac{W_{\mathrm{m}}^{+} T^{+}}{2 \pi} \exp \left(-y^{+} \sqrt{\pi / T^{+}}\right)=122 .
$$

This estimate compares well with a displacement of about 110 wall units, computed by averaging the maximum spanwise movement of 50 streaks. This result is in accordance with the DNS results of Quadrio and Sibilla [4], which show that the space-averaged spanwise flow coincides with the analytic flow solution of the Stokes problem.

The vortical structures centred at $y^{+}=45$ do not seem to be shifted laterally by the transversal Stokes layer. Indeed, a period of oscillation of $T^{+}=67$ generates a Stokes layer of thickness $\delta_{\mathrm{S}}^{+}=\sqrt{4 \pi T^{+}} \approx 30$, location below which only a small portion of the quasi-streamwise population exists [12].

End views were also useful to study the transient behaviour of the flow both in temporal terms, namely over the moving wall from the start-up of the oscillation, and in spatial terms, namely at a location downstream of the oscillating surface where the flow is readjusting to its natural state, but still presents a drag-reducing character. ${ }^{6}$ In the animation of figure 17 , we observe that the near-wall turbulent structure is already, remarkably, affected by the wall motion after only one oscillating cycle. Despite the fact that the turbulence in the proximity of the wall appears strongly modified shortly after the outset of the oscillation, the complete evolution of the wall-shear stress at these oscillating conditions takes up to more than 10 oscillating cycles to reach the new statistically stationary state, as outlined by Quadrio and Ricco [6] for the same oscillatory conditions. This provides more evidence to their view of the wall-shear stress

\footnotetext{
${ }^{6}$ The location of visualization was at about $600 \nu / u_{\tau}$ downstream of the rearmost edge of the moving section, where
} Ricco and $\mathrm{Wu}[14]$ showed that the amount of drag reduction reduces by more than $50 \%$. 


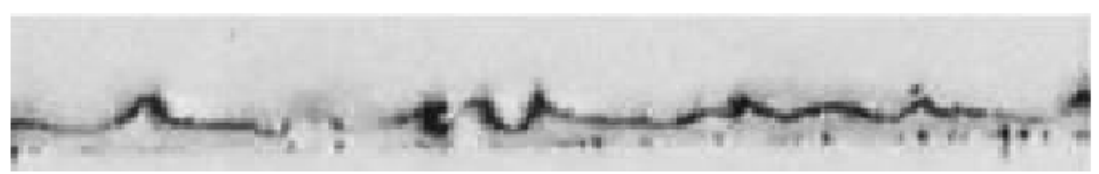

Figure 17. End-view animation for initial transient of the oscillating-wall case $\left(T^{+}=67, D_{\mathrm{m}}^{+}=360\right.$ and $\left.W_{\mathrm{m}}^{+}=17\right)$ with the platinum wire positioned at $y^{+}=25$ and the laser sheet located at a distance of $\Delta x^{+}=240$ downstream of the wire. The total time of the animation is $t^{+}=365$ and the spanwise window is $900 \nu / u_{\tau}$.

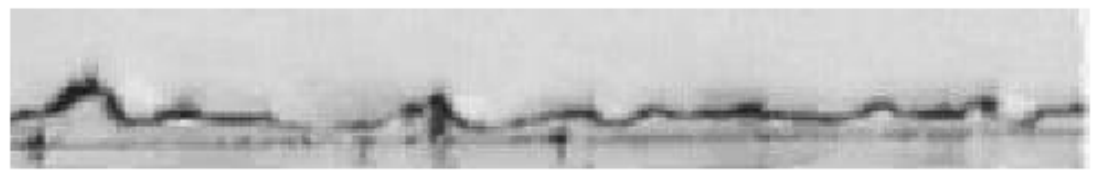

Figure 18. End-view animation of flow downstream of the moving wall, which oscillates according to $T^{+}=67, D_{\mathrm{m}}^{+}=360$ and $W_{\mathrm{m}}^{+}=17$. The platinum wire was positioned at $y^{+}=25$ from the wall and at a distance of $600 \nu / u_{\tau}$ from the rearmost edge of the plate and the laser sheet was located at a distance of $\Delta x^{+}=240$ downstream of the wire. The total time of the animation is $t^{+}=300$.

responding to the changes imposed at the wall in a slower manner than the statistical quantities in the near-wall region.

Downstream of the rearmost edge of the plate, the effect of the oscillation does not vanish forthwith, and the flow presents different features compared with the moving surface (animation of figure 18). Near-wall structures (bubble wire at $y^{+}=25$ ) oscillate in the spanwise direction, whereas the low-speed streaks behave similarly to the unperturbed flow case and are naturally stretched along the streamwise direction, when observed by means of overhead views at the same downstream location. The spanwise oscillation of the low-speed pockets is inevitably put to rest through the no-slip condition at the wall, so that the lateral oscillatory momentum is transferred upward by viscous diffusion and conveyed towards the longitudinal vortices. The modified flow downstream of the moving wall is then similar to the wall-bounded turbulent flow excited by a spanwise oscillating pressure gradient studied by Jung et al [1] and by Akhavan et al [7], where the streamwise vortices are thought to oscillate transversally over the low-speed streaks.

\section{Summary and concluding remarks}

The present work was intended to gain further understanding of the physical mechanisms characterizing wall-bounded turbulent flows subjected to spanwise oscillations of the wall. Hydrogen-bubble flow visualizations were conducted to meet this purpose. The gist of the problem is that the oscillating-wall technique works by effectively reducing both the bursting and the sweeping turbulent activity in the proximity of the wall. The images and animations describe the fully developed state of the new modified flow for values of maximum wall velocities $W_{\mathrm{m}}^{+}<11$, while the visualized cases with higher values of $W_{\mathrm{m}}^{+}$are likely to depict the spatial evolution of the flow from the foremost edge of the oscillating wall section.

The near-wall flow is dragged laterally by the wall oscillation and this results in

- cyclic tilting of streaks with respect to the streamwise direction;

- reduced length of streaks;

- increased spacing of streaks. 
Our study shows that the spacing of streaks increases only for values of drag reduction higher than $25 \%$, while the cyclic tilting of streaks and the reduced length of streaks occur for the entire range of drag-reduction values. The sweeping motions of high-speed fluid from near-wall regions towards the wall are strongly reduced by the oscillating wall since the transversal Stokes layer creates a shield effect against them and largely impedes high-speed fluid to arrive at the wall surface. This mechanism contributes to reduce the wall-friction drag since the sweeping activity is known to be responsible for high wall-shear stress.

The flow shows a fast response to the oscillation, being strongly modified after one oscillating cycle. Downstream of the wall section the flow recovers its natural state by transmitting the transversal oscillatory motion of the low-speed streaks upward to the longitudinal vortical structures by means of viscous diffusion. Structures at higher regions from the wall now oscillate laterally, while the streaks orient in the streamwise direction, similarly to the natural turbulent flow.

\section{Acknowledgments}

The author wishes to thank one of the referees for pointing out reference [7], Dr S Trujillo and Professor D Bogard for their guidance during the experimental analysis and Dr L Casarsa, Dr S Zuccher and M Dixon for proofreading a preliminary version of the manuscript and for the fruitful discussion.

\section{References}

[1] Jung W J, Mangiavacchi N and Akhavan R 1992 Suppression of turbulence in wall-bounded flows by highfrequency spanwise oscillations Phys. Fluids A 4 1605-7

[2] Laadhari F, Skandaji L and Morel R 1994 Turbulence reduction in a boundary layer by local spanwise oscillating surface Phys. Fluids 6 3218-20

[3] Baron A and Quadrio M 1996 Turbulent drag reduction by spanwise wall oscillations Appl. Sci. Res. 55 311-26

[4] Quadrio M and Sibilla S 2000 Numerical simulation of turbulent flow in a pipe oscillating around its axis J. Fluid Mech. 424 217-41

[5] Karniadakis G E and Choi K-S 2003 Mechanisms on transverse motions in turbulent wall flows Ann. Rev. Fluid Mech. 35 45-62

[6] Quadrio M and Ricco P 2003 Initial response of a turbulent channel flow to spanwise oscillation of the walls J. Turbulence 4007

[7] Akhavan R, Jung W and Mangiavacchi N 1993 Control of wall turbulence by high frequency spanwise oscillations AIAA Paper 93-3282

[8] Choi K-S, DeBisschop J R and Clayton B R 1998 Turbulent boundary-layer control by means of spanwise-wall oscillation AIAA J. $\mathbf{3 6} 1157-62$

[9] Choi J-I, Xu C-X and Sung H J 2002 Drag reduction by spanwise wall oscillation in wall-bounded turbulent flows AIAA J. 40 842-50

[10] Iuso G, Di Cicca G M, Onorato M, Spazzini P G and Malvano R 2003 Velocity streak structure modifications induced by flow manipulation Phys. Fluids $\mathbf{1 5}$ 2602-12

[11] Panton R 1997 Self-sustaining Mechanisms of Wall Turbulence (Advances in Fluid Mechanics vol 15)

[12] Robinson S K 1991 Coherent motions in the turbulent boundary layer Ann. Rev. Fluid Mech. 23 601-39

[13] Coughran M T 1988 Interdependence of large and small structures in a turbulent boundary layer PhD Thesis The University of Texas at Austin

[14] Ricco P and Wu S 2004 On the effects of lateral wall oscillations on a turbulent boundary layer Exp. Therm. Fluid Sci. in press

[15] Kays W M and Crawford M E 1993 Convective Heat and Mass Transfer 3rd edition (New York: McGraw-Hill)

[16] Mueller T J 1983 Flow visualization by direct injection Fluid Mechanics Measurements ed R J Goldstein (New York: Hemisphere) 
Modification of near-wall turbulence due to spanwise wall oscillations

[17] Lu L J and Smith C R 1991 Use of flow visualization data to examine spatial-temporal velocity and burst-type characteristics in a turbulent boundary layer J. Fluid Mech. 232 303-40

[18] Oldaker D K and Tiederman W G 1977 Spatial structure of the viscous sublayer in drag-reducing channel flows Phys. Fluids 20 133-44

[19] Tiederman W G, Luchik T S and Bogard D G 1985 Wall-layer structure and drag reduction J. Fluid Mech. 156 419-37

[20] Schlichting H and Gersten K 2000 Boundary-Layer Theory (Berlin: Springer)

[21] DeGraaff D B and Eaton J K 2000 Reynolds-number scaling of the flat-plate turbulent boundary layer J. Fluid Mech. 422319

[22] Kline S J, Reynolds W C, Schraub F A and Runstadler P W 1967 The structure of turbulent boundary layers J. Fluid Mech. 30741

[23] Kim H T, Kline S J and Reynolds W C 1971 The production of turbulence near a smooth wall in a turbulent boundary layer J. Fluid Mech. 50 133-60

[24] Smith C R and Metzler S P 1983 The characteristics of low-speed streaks in the near wall region of a turbulent boundary layer J. Fluid Mech. 129 27-54

[25] Krogstad P A, Kaspersen J H and Rimestad S 1998 Convection velocities in a turbulent boundary layer Phys. Fluids 10 949-57

[26] Quadrio M and Ricco P 2004 Critical assessment of turbulent drag reduction through spanwise wall oscillations J. Fluid Mech. (submitted for publication)

[27] Achia B U and Thompson D W 1977 Structure of the turbulent boundary in drag-reducing pipe flow J. Fluid Mech. 81 439-64

[28] Donohue G L, Tiederman W G and Reischman M M 1972 Flow visualization of the near-wall region in a drag-reducing channel flow J. Fluid Mech. 56 559-75 